\title{
Transfusion Medicine and Hemotherapy
}

\section{Possible Measures for Reduction the Potential Risk of vCJD Transmission by Blood and Blood Products}

\section{Measures for the Optimal Use of Blood Products}

Blood products are an essential component of any modern clinical treatment. Being 'medicinal products from humans', they cannot be entirely risk-free, despite the great progress in safety. Critical indication and restrictive administration of blood products are therefore essential tools that reduce the residual risk, which is also true for a potential transmission of vCJD by donor blood.

Examples of barely scientifically justifiable differences in the indication of blood transfusion can be found in the Sanguis Study [89, 90]. In the extreme case, the preoperative request for provision of RBCC in cholecystectomy was more than 10 times that of the actually transfused units. Noticeable differences were found in the frequency of transfusion among 43 hospitals participating in the studies from 10 European countries. For hemicolectomies, the range was between 0 and $79 \%$ of the patients; such differences cannot be explained by differences between the patient groups studied. This heterogeneous transfusion practice has not changed significantly in the past few years: a more recent Finnish study thus shows that, contrary to international recommendation, the median of pretransfusional hemoglobin $(\mathrm{Hb})$ values in transurethral prostate resection was $112 \mathrm{~g} \mathrm{Hb} / \mathrm{l}$ [91]. Various authors have stated unanimously that prospective determination of administration criteria and consistent instructions of personnel would lead to a considerable reduction of the consumption of blood components [92, 93].

A possible approach to avoiding potential risks relating to (allogeneic) blood products prepared from donors would be treatment of patients by autologous blood transfusion. The risk of a new infection with blood-associated pathogens (including vCJD) is excluded in autologous blood treatment. Such autologous blood transfusions, however, can be performed only in elective surgery with a timely, sufficiently and reliably foreseeable transfusion requirement. The Arbeitskreis Blut has currently made a statement on the applicability and the importance of autologous blood treatment [94].

Under German EU presidency in 1999, a meeting was held in Wildbad Kreuth [95] with experts in attendance from the EU member states. During this meeting, an assessment of the current situation concerning the use of the most important blood products was elaborated, and central questions of critically assessed use, quality management, and economic aspects of transfusion medicine were summarized. It would be desirable to continue the initiative of Wildbad Kreuth.

In regards to therapy with blood components and plasma derivatives an interdisciplinary working group of the Bundesärztekammer summarized basic principles for a clinically indicated use of all important blood products with special consideration of the international literature, national and international consensus conferences and clinical experiences, and has made this publication accessible to all centers of transfusion medicine in Germany [96]. At a European level, too, appropriate recommendations were adopted on hemotherapy [97].

There has been increased awareness of this problem in the past few years. An essential contribution for this has been the requirement laid down in the Transfusion Act (Transfusionsgesetz; TFG) of July 1, 1998 for the establishment of a well-functioning quality assurance system for the use of blood products in health care facilities. According to the TFG, these health care facilities must employ appropriately qualified physicians as persons responsible for transfusion and, in addition, transfusion representatives in each clinical unit. For this purpose, the Professional Organization of German Transfusionists (Berufsverband Deutscher Transfusionsmediziner e. V.) has developed a model quality management handbook that is regularly updated, as are the guidelines and guidances of the Bundesärztekammer.

The requirements for such a quality assurance system were transposed into the hemotherapy guidelines [75] where they were further specified. Of crucial importance will be the way in which these guidelines are implemented and used by hospitals and doctors, efforts of which must not decline.

It is necessary that such efforts are actively encouraged on the part of the top managers of health care facilities and by the health policy makers and that they are recognized by the health care providers who are supposed to finance them. We must demand the further development and implementation of guidelines and guidances and their transposition into well-functioning systems for 
quality assurance in the use of blood products in health care facilities as an essential contribution to safety, particularly in an effort to avoid a potential vCJD transmission.

The demand for an optimal use of blood and blood products has still not lost its justification. This requirement is undisputed, especially as a safety measure in view of vCJD. Shortly after the first suspected case of clinical transmission was disclosed, the EU Commission called in a 'Technical Meeting of Blood Experts related to vCJD transmission' on January 20, 2004 in Luxembourg. One of the statements in the paper elaborated by this meeting reads as follows:

'There was agreement that optimal use of blood may further reduce the risk of transmission of vCJD by avoiding unnecessary exposure to allogeneic blood transfusion. In addition avoiding unnecessary transfusion may improve the availability of blood for transfusion; this in turn may facilitate the introduction by Member States of additional donor deferrals if required.'

\section{Diagnosing vCJD: Screening Tests}

When it comes to diagnosing vCJD infection or the disease itself, a distinction must be made between screening tests on one hand and tests for clinical diagnosis on the other. The development of screening tests for vCJD is being intensively pursued by a number of groups, but so far no concrete success has emerged from any of the various approaches. The principal goal of a screening test is to detect vCJD infections as early as possible before onset of initial symptoms in order to prevent possible further transmissions and, if appropriate, to allow therapeutic measures to be taken in an appropriate time frame.

Clinical diagnosis, in contrast, is carried out on patients who are already displaying symptoms of the disease. In this case it is a question of investigating the suspicion of vCJD or another neurological disease based on various parameters. Definitive diagnosis of vCJD can in principle be carried out in live patients by screening for $\mathrm{PrP}^{\mathrm{Sc}}$ in the tonsils $[28,40]$. However, a biopsy that presents such a burden for the patient would only, if at all, be performed in the case of serious suspicion. At present, however, a confirmation of vCJD infection by histological display of the amyloid plaque or detection of the $\mathrm{PrP}^{\mathrm{Sc}}$ in brain material by Western blot is only possible after the death of the patient. Clinical diagnosis is a laborious process consisting of various methods and is of subordinate importance for the safety of blood donations. A summary may be found in 'Appendix (C) Diagnosing CJD' (pp 29).

The development of screening tests is one of the key endeavours for the safety of blood donations, especially if the testing of blood donors with a sensitive test could ensure the direct detection of persons infected with vCJD who are still in the incubation period. Such a test would be superior to indirect measures such as the prophylactic exclusion of groups of donors who had been exposed to a higher theoretical risk of vCJD.

The approaches currently being pursued for screening tests are based either on direct detection of the pathological prion protein
$\left(\mathrm{PrP}^{\mathrm{Sc}}\right)$, which is generally believed to correlate with TSE infectivity, in blood or other easily accessible body fluids or on the detection of other markers associated with the infection (surrogate markers).

At present, a range of tests are under development [98], but data on sensitivity and specificity are as yet largely absent. One of the problems in the detection of the $\mathrm{PrP}^{\mathrm{Sc}}$ protein in body fluids is the extremely low concentration at which it naturally occurs in the periphery, if at all. The data available so far allow us to draw the analogue conclusion that for the task of detecting vCJD in human blood the sensitivity limits of currently known test systems will quickly be reached. Estimates expect considerably less than $1 \mathrm{pg} / \mathrm{ml}$ $\mathrm{PrP}^{\mathrm{Sc}}$ in the blood. The most sensitive antigen tests (e.g. for the detection of HBsAg of HBV or p24 of HIV, two proteins with a molecular size similar to that of $\mathrm{PrP}^{\mathrm{Sc}}$ ), after many years of development and improvement, are capable of detecting antigen only at levels of $10 \mathrm{pg}$ and above per milliliter plasma or serum. In addition, physiological prion protein is present in approximately 10,000-fold excess, which makes the sensitive and specific detection of $\mathrm{PrP}^{\mathrm{Sc}}$ considerably more difficult. Highly specific so-called 'conformational' antibodies (for the recognition of $\mathrm{PrP}^{\mathrm{Sc}}$ characteristic folding epitopes or conformation epitopes) therefore seem indispensable for a sensitive detection of this protein. The possible use of such an immunoassay (CDI; 'conformation dependent immunoassay') for clinical diagnostics of human TSE infections is currently under discussion [99].

Current research projects designed to establish a $\operatorname{PrP}^{\mathrm{Sc}}$ screening test are pursuing different approaches to surmounting these limitations, e.g. attempts to increase the tests sensitivity by means of spectroscopic techniques. Other approaches use enrichment steps to increase the $\operatorname{PrP}^{\mathrm{Sc}}$ concentration in the sample to be tested by selective precipitation of $\mathrm{PrP}^{\mathrm{Sc}}$ through its binding to 'ligand' molecules or cyclical amplification of the pathogen prion protein. The artificial in vitro replication of $\mathrm{PrP}^{\mathrm{Sc}}$ by means of the PMCA (protein misfolding cyclic amplification) method [100] has especially raised high expectations. However, despite demonstration of the possibility to replicate infectious $\mathrm{PrP}^{\mathrm{Sc}}$ in one species by a factor of $10^{3}[98,101]$, this has not yet led to the development of appropriate test systems.

The obvious difficulties with the sensitive detection of the $\operatorname{PrP}^{\mathrm{Sc}}$ prion protein, the only known specific marker of vCJD infection, have led to the exploration of alternative test concepts. A possible choice would be a screening method that allows us to use one or more markers which, alone or in combination, would permit a reliable detection of vCJD (surrogate markers). Such a screening procedure could be carried out both at the RNA level (differential display) and at the protein level (proteome analysis).

Previous analyses of the modified regulation of genes in TSE infections (differential gene expression) have shown that a number of genes are over- or under-expressed in the course of the disease. In the past few years, several working groups have examined to what extent the differential expression of genes in the course of the disease can contribute to a better understanding of the infection. In addition to a number of genes already identified which are up- or 
down-regulated during a prion disease, much attention has been paid to the publication of a peripheral marker detectable in blood cells (erythroid differentiation factor; EDF) [102]. Follow-up tests, however, showed that this marker is subject to major fluctuations in healthy individuals [103]. Based on the intensive work done in this field, several surrogate markers have been published to be candidates for screening markers while proof for this remains to be provided.

Extensive examinations on well-defined populations and acceptable test features (sensitivity, specificity, high throughput) are indispensable preconditions for introducing a screening test, especially if it is to be used in blood donation screening. These conditions have so far not been met by any of the test procedures discussed in the literature. The question arises as to what extent the criteria should be established for validation. Evaluating new tests in healthy populations, e. g. blood donors, raises a number of unresolved ethical questions, such as how reactive test results are to be handled which, at least when obtained with the first available test, cannot be confirmed or clarified by another method.

\section{Exclusion of Certain Categories of Persons from Donating Blood}

As explained in 'Diagnosing vCJD: Screening Tests' (see above), it is not foreseeable whether and when a routine test suitable for blood donor screening will be available that could reliably detect the agent in humans in the preclinical phase in which the pathogen concentration is probably still particularly low. The effectivity of manufacturing steps suitable to reduce potential infectivity in blood components for transfusion (e.g. prion-adsorbing blood filters) cannot currently be assessed either.

Therefore, donor selection criteria based on the history of the donor must in principle still be used for risk prevention. Such criteria have been an important part of precautionary measures for many years. An overview can be found in 'Appendix (E) Exclusion of Transfusion Recipients: Estimating the Consequences' (pp 34). Development and justification of the donor exclusion criteria established some years ago were described in the previous report of the working group in 2001. On May 7, 2005 the Paul-Ehrlich-Institut ordered that individuals operated on or transfused in the UK must to be excluded from donating blood. In addition, this announcement required that reference to the transmission cases observed should be made in the package leaflet and the Summary of Product Characteristics (SPC). The regulations applicable in Germany have been adopted in the hemotherapy guidelines of the Bundesärztekammer and Paul-Ehrlich-Institut [75].

Other important questions discussed in the past few years relate to whether the potential transmission by transfusion could lead to a perpetuation of vCJD among humans, even though transmission through the food chain has been stopped, and whether an exclusion of transfusion recipients could essentially influence the course of the vCJD epidemic. In several European countries, including the UK, The Netherlands, Switzerland and France - there albeit as early as 1998 under the impression of virus transmissions -, the exclusion of transfusion recipients from donating blood has meanwhile been laid down with the aim of preventing the further spread of the vCJD agent by this route. In its announcement of April 29, 2004, the Paul-Ehrlich-Institut began a graduated plan.

In order to have a scientific database for the decision, a model calculation was prepared as suggested by the working group shown in 'Appendix (D) Model Calculation Addressing an Exclusion of Donors with a History of Transfusion' (pp 32). The calculations are based on pessimistic assumptions. Concerning the number of individuals in Germany expected to be infected with the vCJD pathogen and potentially able to transfer this pathogen, the assumed number of 2,000 within 10 years even exceeds the estimate of the 2001 report, which today seems very pessimistic. The same is true concerning the assumed duration of the incubation period and the assumption that any contaminated blood transfusion would transmit vCJD with $100 \%$ effectivity. For a correct consideration of demographic structures, authentic data were collected of donor and recipient populations. Even though it must be anticipated that there will be vCJD transmissions by transfusion, it must also be expected that the overwhelming majority will be caused by donors infected by consumption of BSE-contaminated food. The results show that excluding transfusion recipients will not essentially change the epidemiological outcome, and even the effect of prevention in isolated cases would be minimal at best.

However, the planned exclusion of transfusion recipients would have entailed serious problems and disadvantages. The French example shows that such exclusion is possible in principle but involves major problems and efforts and can therefore be implemented only over a longer period of time. To secure the provision of blood supplies, new donors would have to be recruited to a considerable extent. In this context, it must be considered that, according to the data on epidemiology collected by the Robert Koch-Institut pursuant to Section $22 \mathrm{TFG}$, the prevalence of virus infections in new blood donors is higher than in long-term donors. Another problem is that exclusion of transfusion recipients could be perceived as a signal that, despite all efforts, blood supplies are not sufficiently safe.

In conclusion, the following can be stated: the intended measure, i. e. exclusion of transfusion recipients for the prevention of a further spread of vCJD by blood transfusions would bring about only a marginal effect, taking into account the epidemiological situation and demographic structures in Germany. The measure would, on the other hand, involve considerable disadvantages. On the basis of the present assessment of the situation, one would refrain from the measure announced on April 29, 2004. 